\title{
Phosphate Binder, Ferric Citrate, Attenuates Anemia, Renal Dysfunction, Oxidative Stress, Inflammation, and Fibrosis in 5/6 Nephrectomized CKD Rats
}

\author{
Wanghui Jing, Ane C. F. Nunes, Ted Farzaneh, Mahyar Khazaeli, Wei Ling Lau, \\ and Nosratola D. Vaziri \\ School of Pharmacy, Xi'an Jiaotong University, Xi'an, PR China (W.J.); and Division of Nephrology and Hypertension, Department \\ of Physiology and Biophysics (W.J., A.C.F.N., M.K., W.L.L., N.D.V.) and Department of Pathology and Laboratory Medicine (T.F.), \\ University of California, Irvine, California
}

Received April 13, 2018; accepted August 1, 2018

\begin{abstract}
Chronic kidney disease (CKD) causes anemia and impairs intestinal iron absorption. However, use of the phosphate binder ferric citrate (FC) increases body iron stores and hemoglobin levels in CKD patients. By intensifying oxidative stress and inflammation iron overload resulting from excessive use of intravenous iron can accelerate CKD progression. The present study explored the route of absorption and tissue distribution of iron with FC administration and its effect on renal function, histology, and inflammatory, oxidative, and fibrosis pathways in CKD rats. Male Sprague Dawley rats were randomized to sham-operated control (CTL) group and 5/6 nephrectomized (CKD) groups fed either regular or 4\% FC-supplemented diets for 6 weeks. Animals were then sacrificed, and blood and target tissues were harvested and processed. The untreated CKD rats
\end{abstract}

exhibited anemia, hypertension, upregulation of renal tissue inflammatory, oxidative, and fibrotic pathways, impaired nuclear translocation, and downregulation of Nrf2's target gene products and depletion of colonic epithelial tight junction proteins. FC administration raised serum iron, improved anemia, attenuated hyperphosphatemia, partially improved renal function, reduced oxidative stress, inflammation, and fibrosis, and restored colonic epithelial zonula occludens-1 protein abundance. Tissue iron staining detected presence of iron in epithelial cells and subepithelium of colon and in renal proximal tubules. In conclusion ferric citrate administration resulted in modest amelioration of renal function and histology and partial improvements of fibrosis, inflammation, and oxidative stress in the kidney tissues of CKD rats.

\section{Introduction}

Nearly all end-stage renal disease (ESRD) patients and 70\% of patients with earlier stages of chronic kidney disease (CKD) have anemia (McFarlane et al., 2008), and iron deficiency is a major contributing cause of anemia in the CKD population. Numerous factors contribute to iron deficiency in CKD patients, including surgical procedures for vascular access, frequent blood drawing for laboratory tests, and blood loss into the hemodialysis apparatus (Kalantar-Zadeh et al., 2009). In addition, intestinal absorption of iron is suppressed as a result of systemic inflammation-induced elevation of hepcidin (Vaziri et al., 2016a). Furthermore, owing to the following disorders, routine use of recombinant erythropoiesis-stimulating agents (ESA) frequently

This work was financially supported, in part, by the International Postdoctoral Exchange Fellowship Program [No. 20150050] and National Natural Science Foundation of China [No: 81603370]. ACFN received research grant from $\mathrm{CNPq} /$ Brazil, Science Without Borders Program.

https://doi.org/10.1124/jpet.118.249961. leads to iron deficiency: 1) extensive transfer of iron from the storage sites to erythroid progenitor cells (Eschbach et al., 1987) and 2) impaired mobilization of iron from storage sites, which leads to functional iron deficiency.

Iron supplementation is a vital component of anemia treatment in patients with CKD, including oral and intravenous (IV) administration. Because intestinal absorption of iron is impaired, oral iron compounds are generally less effective than IV preparations in advanced CKD patients. However, the indiscriminate use of IV iron can lead to side effects that cannot be detected by short-term clinical trials. Use of IV iron preparations can lead to iron overload. At physiologic concentrations, iron is kept in a catalytically inactive state by various binding proteins. However, the unliganded iron can trigger oxidative stress by converting $\mathrm{H}_{2} \mathrm{O}_{2}$, which is abundantly generated in mitochondria, to hydroxyl radical $(\cdot \mathrm{OH})$, the most reactive oxygen radical $\left(\mathrm{H}_{2} \mathrm{O}_{2}+\mathrm{Fe}^{2+} \rightarrow \cdot \mathrm{OH}+\mathrm{OH}^{-}+\mathrm{Fe}^{3+}\right)$. Mitochondria and mono-oxygenase enzymes produce superoxide, which—by

ABBREVIATIONS: CKD, chronic kidney disease; COX-2, cyclooxygenase-2; Cu/Zn-SOD, copper-zinc superoxide dismutase; CTL, control group; eNOS, endothelial nitric oxide synthase; ESA, erythropoiesis-stimulating agents; ESRD, end-stage renal disease; FC, ferric citrate; iNOS, inducible nitric oxide synthase; IV, intravenous; JAM-1, junctional adhesion molecule-1; MCP-1, monocyte chemotactic protein 1; MPO, myeloperoxidase; $\mathrm{NF}-\kappa \mathrm{B}$, nuclear factor- $\kappa \mathrm{B}$ p65; Nrf2, nuclear factor erythroid 2-related factor 2; PAl-1, plasminogen activator inhibitor-1; $\alpha$-SM actin, $\alpha$-smooth muscle actin; TGF- $\beta$, transforming growth factor- $\beta$; ZO-1, zonula occludens 1 . 
TABLE 1

General data

Data are mean \pm S.E.M.

\begin{tabular}{lrrc}
\hline & CTL $(n=9)$ & CKD $(n=9)$ & CKD+FC $(n=9)$ \\
\hline SBP (mm Hg) & $111.2 \pm 3.6$ & $145.4 \pm 2.1^{*}$ & $111.4 \pm 4.1^{\#}$ \\
Hemoglobin (d/dl) & $13.4 \pm 0.1$ & $11.4 \pm 0.5^{*}$ & $13.7 \pm 0.1^{\#}$ \\
BUN (mg/dl) & $18.6 \pm 1.9$ & $41.9 \pm 3.5^{*}$ & $36.8 \pm 5.1^{*}, \#$ \\
24 h urine total protein & $11.1 \pm 0.9$ & $37.0 \pm 1.9^{*}$ & $30.8 \pm 2.9^{*}$ \\
$\quad(\mathrm{mg} / \mathrm{dl})$ & & & \\
Serum creatinine & $0.36 \pm 0.1$ & $0.77 \pm 0.1^{*}$ & $0.61 \pm 0.1^{*, \#}$ \\
$\quad(\mathrm{mg} / \mathrm{dl})$ & & & \\
Creatinine clearance & $1.49 \pm 0.5$ & $0.67 \pm 0.2^{*}$ & $0.91 \pm 0.2^{*}, \#$ \\
$\quad(\mathrm{ml} / \mathrm{min})$ & & & \\
Serum iron ( $\mu \mathrm{g} / \mathrm{dl})$ & $224.6 \pm 25.2$ & $167.1 \pm 10.7^{*}$ & $191.4 \pm 26.7^{\#}$ \\
$\begin{array}{l}\text { Serum calcium (mg/dl) } \\
\text { Serum phosphate }\end{array}$ & $9.8 \pm 0.9$ & $10.1 \pm 1.5$ & $10.1 \pm 0.6$ \\
$\quad 8.6 \pm 0.3$ & $12.4 \pm 0.1^{*}$ & $8.8 \pm 0.9^{\#}$ \\
$\quad(\mathrm{mg} / \mathrm{dl})$
\end{tabular}

BUN, blood urea nitrogen; FC, $4 \%$ ferric citrate in diet; SBP, systolic blood pressure.

$* P<0.05$ vs. CTL ${ }^{\#} P<0.05$ vs. CKD.

converting $\mathrm{Fe}^{3+}$ to $\mathrm{Fe}^{2+}$ (Haber-Weiss reaction)—mediates continuous production of $\mathrm{OH}$ and intensification of oxidative stress. Several in vitro and in vivo studies have shown that excessive use of IV iron preparations can increase the risk of infection (Brookhart et al., 2013; Agarwal et al., 2015), cause oxidative stress (Salahudeen et al., 2001; Tovbin et al., 2002; Lim and Vaziri, 2004a,b; Pai et al., 2007; Kuo et al., 2008; Garcia-Fernandez et al., 2010), and lead to iron overload (Ferrari et al., 2011; Vaziri, 2012).

Earlier studies have demonstrated the contribution of intestinal microbial dysbiosis and disruption of colonic epithelial junctional complex to the systemic inflammation in humans and animals with CKD (Wong et al., 2006; Vaziri et al., 2012, 2013a,b,c, 2016b). Use of the ferric citrate (FC) complex, which is an iron-containing phosphate binder, increases body iron stores, raises hemoglobin level, and reduces the need for ESA and IV iron in ESRD and CKD patients (Block et al., 2015; Lewis et al., 2015). A recent phase 3 clinical trial demonstrated the safety and efficacy of ferric citrate for treatment of iron deficiency anemia in dialysisindependent CKD patients (Fishbane et al., 2017), with the adverse side effects of the drug in this trial limited to gastrointestinal disorders. In contrast to the IV iron, which has been shown to intensify oxidative stress (Tovbin et al., 2002; Lim and Vaziri, 2004a; Kuo et al., 2008), restoration of iron stores with ferric citrate administration did not raise markers of oxidative stress and inflammation in Japanese hemodialysis patients (Tanaka et al., 2016). In fact, ferric citrate administration resulted in a mild but insignificant decrease in markers of inflammation and oxidative stress (Tanaka et al., 2016).

The current study was designed to determine the effect of clinically relevant dosages of ferric citrate on renal structure and function, biomarkers of oxidative stress, inflammation, and fibrosis, colonic epithelial barrier, as well as the route of iron absorption and tissue distribution in CKD rats.

\section{Materials and Methods}

Study Groups. Eight-week-old male Sprague-Dawley rats ( 250 g) were purchased from Charles River Laboratories (Raleigh, NC). Animals were housed in a climate-controlled vivarium with 12-hour day/night cycles and provided food and water ad libitum. The rats underwent sham surgery or 5/6 nephrectomy and after 1 week the animals were randomized into three groups: sham-operated control group (CTL), CKD rats fed regular diet (CKD), and CKD rats provided food containing $4 \%$ ferric citrate $(\mathrm{CKD}+\mathrm{FC})$ for 6 weeks.

The dosage of ferric citrate employed in the present study was chosen on the basis of an earlier dose-response study that had identified the diet containing $3 \%$ ferric citrate as the optimal dosage in normal rats (Matsuo et al., 2014). Since the average food intake in CKD rats (12 g/day) was significantly lower than control rats (18 g/day), we used $4 \%$ ferric citrate in our study to match the previously demonstrated optimal drug dosage. Food intake was similar in the untreated and FC-treated CKD rats.

The rats were placed in metabolic cages for a 24-hour urine collection at the beginning and the final week of the study period. Systolic blood pressure was measured by tail plethysmography as described in our previous report (Vaziri et al., 2002). Ferric citrate was obtained from Keryx Inc. (Boston, MA). For surgical procedures and euthanasia, rats were placed into a sealed anesthesia induction chamber under 5\% isoflurane (Piramal Clinical Care, Bethlehem, $\mathrm{PA}) /$ oxygen gaseous mixture to induce sedation and maintained at $2 \%-4 \%$. Before starting 5/6 nephrectomy procedures, all rats received $0.05 \mathrm{mg} / \mathrm{kg}$ buprenex (Reckitt Benckiser Pharmaceutical Inc., Richmond, VA) for pain relief. The rats were euthanized by cardiac exsanguination, and kidney, liver, proximal jejunum, and descending colon were immediately obtained and processed for analysis. All experiments were approved by University of California, Irvine Institutional Committee for the Use and Care of Experimental Animals. Serum urea was measured by QuantiChrom Urea Assay Kit (BioAssay Systems, Hayward, CA). Serum and urinary creatinine were measured by QuantiChrom Creatinine Assay Kit (BioAssay Systems).

Histologic Analysis. Immediately after sacrifice, kidneys were resected and fixed in 10\% buffered formalin solution. Histologic sections were stained with hematoxylin and eosin (H\&E), and Masson trichrome and blindly evaluated and scored by the pathologist coinvestigator. The glomerular histopathology was graded into five categories: 0 (no sclerosis), 1 (less than $25 \%$ sclerosis), 2 (25\%-50\% sclerosis), 3 (50\%-75\% sclerosis), and 4 (75\%-100\% sclerosis). The glomerulosclerosis index score was calculated as follows: $1 \times$ number of glomeruli with grade $1+2 \times$ number of glomeruli with grade $2+$ $3 \times$ number of glomeruli with grade $3+4 \times$ number of glomeruli with grade $4 /$ total number of counted glomeruli. Approximately 50-60 glomeruli were examined in each animal. Tubulointerstitial lesion was graded on the basis of the extension of inflammatory cell infiltration, fibrosis, tubular dilatation, and atrophy as follows: 0 (normal), grade $1(<10 \%)$, grade $2(10 \%-25 \%)$, grade $3(25 \%-50 \%)$, grade $4(50 \%-75 \%)$, and grade $5(75 \%-100 \%)$.

Tissue Iron Deposition. Iron deposition was determined in renal cortex, liver, proximal jejunum, and descending colon using Prussian Blue staining. Ten sequential photos were taken per sample at $200 \times$ magnification and used to quantify the iron deposits in each tissue using the ImageJ software (U.S. National Institutes of Health, Bethesda, MD, https://imagej.nih.gov/ij/).

Western Blot Analyses. Cytoplasmic and nuclear extracts from the renal and colon tissues were obtained as described previously (Sakurai et al., 1996; Vaziri et al., 2013b). The target proteins in the kidney and colon tissues were measured by Western blot analysis, as described previously (Eschbach et al., 1987; Kim and Vaziri, 2010; Aminzadeh et al., 2012), using the following antibodies: Rabbit antibodies against rat nuclear factor- $\kappa \mathrm{B}$ p65 (NF- $\kappa \mathrm{B}$ p65) (cat. no. ab16502, $60 \mathrm{kDa}$ ), monocyte chemotactic protein 1 (MCP-1) (cat. no. ab25124, $25 \mathrm{kDa}$ ), inducible nitric oxide synthase (iNOS) (cat. no. ab3523, $135 \mathrm{kDa}$ ), NADPH-oxidase 4 (NOX4) (cat. no. ab133303, $67 \mathrm{kDa}$ ), gp91 ${ }^{\text {phox }}$ (cat. no. ab129068, $65 \mathrm{kDa}$ ), nitrotyrosine (cat. no. ab183391), Nrf-2 (cat. no. ab89443, $68 \mathrm{kDa}$ ), copper-zinc superoxide dismutase (Cu/Zn-SOD) (cat. no. ab13498, $18 \mathrm{kDa}$ ), cyclooxygenase-2 (COX-2) (cat. no. ab15191, $69 \mathrm{kDa}$ ), heme oxygenase-1 (HO-1) (cat. no. ab13248, $32 \mathrm{kDa}$ ), catalase (cat. no. ab76110, $60 \mathrm{kDa}$ ), myeloperoxidase (MPO) (cat. no. ab65871, $84 \mathrm{kDa}$ ), endothelial nitric oxide 


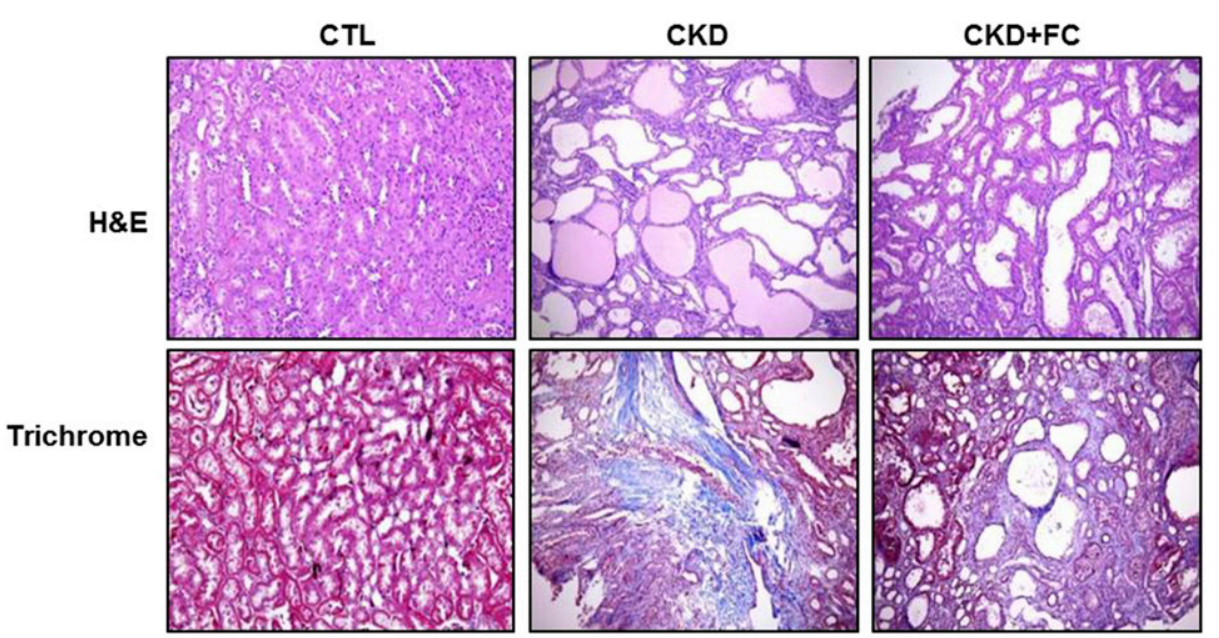

Tubulointerstitial Injury

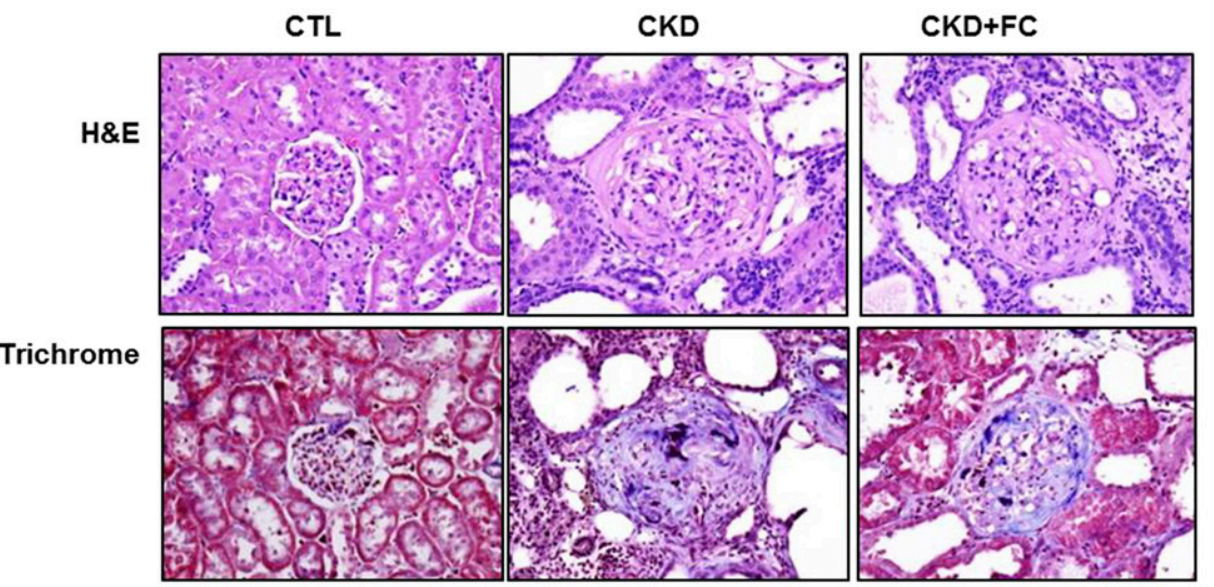

Glomerulosclerosis
Fig. 1. Representative photomicrographs of the H\&E- and Trichrome-stained kidney sections of kidney tissues from CTL and CKD- and CKD+FC-treated animals. Data depicting glomerulosclerosis index and tubulointerstitial injury in different groups. Data are mean \pm S.E.M. $(n=9) . * P<0.05$; $*^{*} P<0.01$.
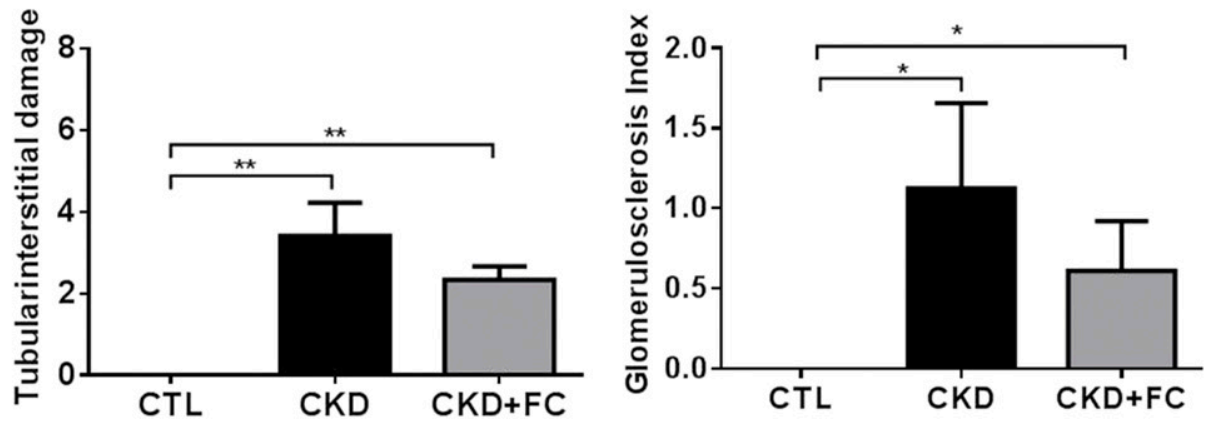

synthase (eNOS) (cat. no. ab95254, $133 \mathrm{kDa}$ ), transforming growth factor- $\beta$ (TGF- $\beta$ ) (cat. no. ab92486, $44 \mathrm{kDa}$ ), plasminogen activator inhibitor-1 (PAI-1) (cat. no. ab66705, $45 \mathrm{kDa}$ ), $\alpha$-smooth muscle actin ( $\alpha$-SM actin) (cat. no. ab5694, $42 \mathrm{kDa}$ ), zonula occludens 1 (ZO-1, cat. no. ab190085, $195 \mathrm{kDa}$ ), junctional adhesion molecule-1 (JAM-1, cat. no.ab52647, $33 \mathrm{kDa}$ ), and occludin (cat. no.ab167161, $59 \mathrm{kDa}$ ) were purchased from Abcam (Cambridge, MA). Antibody against glutathione peroxidase (cat. no. AF3798, $22 \mathrm{kDa}$ ) was purchased from R\&D Systems (Minneapolis, MN). Mouse antibodies against histone H3 (cat. no. ab32356, $17 \mathrm{kDa}$ ) and GAPDH (cat. no. ab8245, $36 \mathrm{kDa}$ ) purchased from Abcam were used to measure housekeeping proteins for nuclear and cytosolic target proteins, respectively.

Statistical Analysis. Data are presented as mean \pm S.E.M. Oneway analysis of variance and multiple comparisons were performed using GraphPad Prism 6.0 (GraphPad Software, San Diego, CA). Tukey's post-test was used to determine differences between the groups. $P$ values less than 0.05 were considered significant.

\section{Results}

General Data. Compared with the control group, the untreated $\mathrm{CKD}$ rats showed significant increases in arterial blood pressure (1.3-fold) and blood urea nitrogen (BUN) (2.3fold), serum creatinine (2.1-fold) and phosphate concentrations (1.4-fold), and urinary protein excretion (3.4-fold) (Table 1). This was associated with significant decreases in creatinine clearance $(0.4$-fold) and hemoglobin concentration 


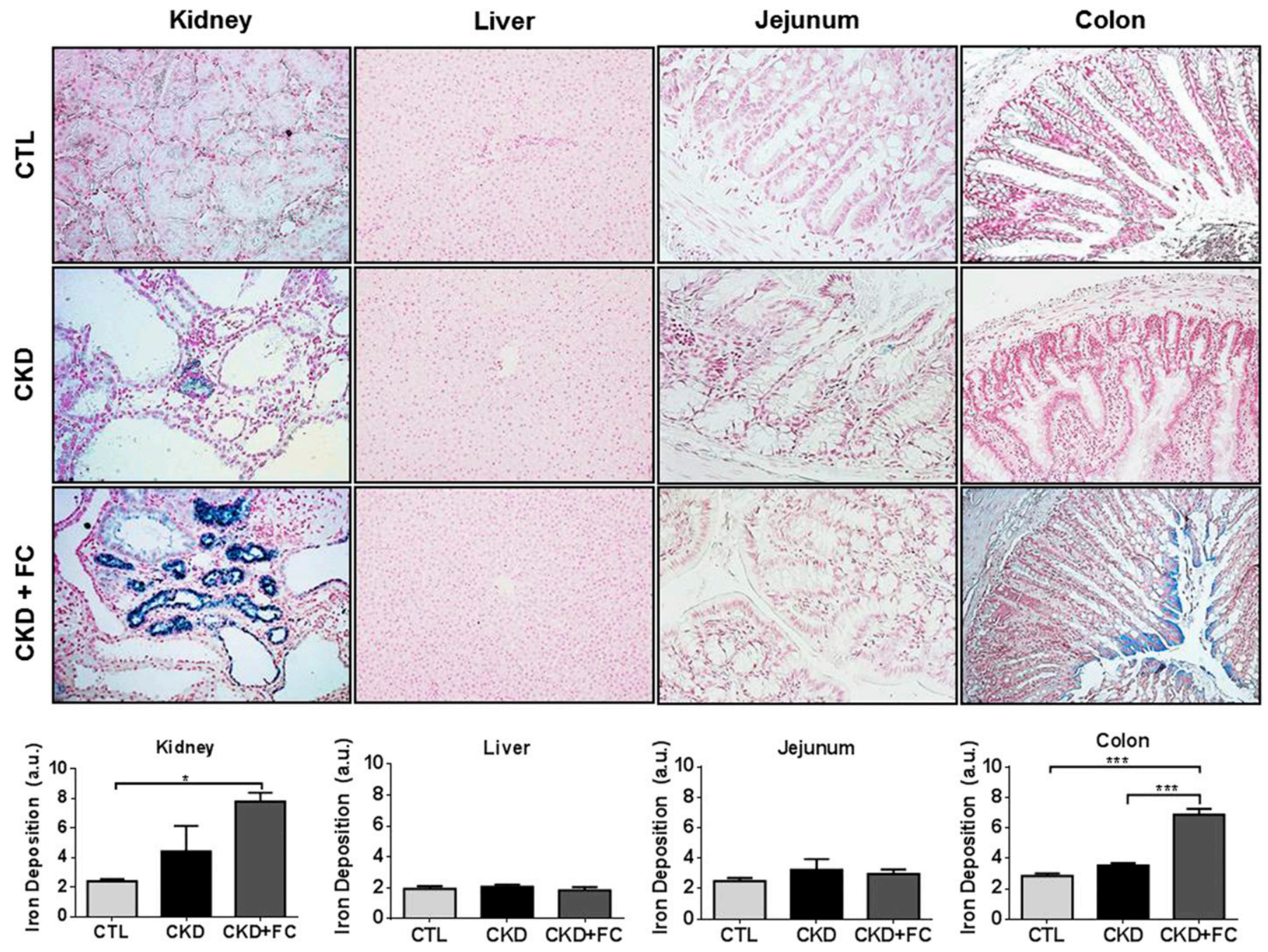

Fig. 2. Representative photomicrographs of kidney, liver, proximal jejunum, and descending colon from CTL and CKD- and CKD+FC-treated animals. Tissues were stained with Prussian Blue for iron detection and counterstained with hematoxylin. Images taken at $200 \times$ magnification were used to identify localization of the iron deposits and quantified by ImageJ software using arbitrary unit (a.u.). Data are mean \pm S.E.M. $(n=9)$. $* P<0.05$; *** $P<0.001$.

(0.8-fold). Compared with the CKD rats, the FC-treated CKD group showed significantly lower blood pressure (0.8-fold), BUN (0.9-fold), and serum phosphate (0.7-fold), and creatinine ( 0.8 -fold) levels and higher creatinine clearance (1.4-fold), serum iron (1.1-fold), and hemoglobin (1.2-fold) concentrations.

Kidney Histology. The weight of the remnant kidneys in the ferric citrate-treated CKD group $(1.27 \pm 0.51 \mathrm{~g})$ was significantly lower than that measured in the untreated CKD rats $(2.87 \pm 0.85 \mathrm{~g})$ reflecting amelioration of renal mass reduction-induced hypertrophy and fibrosis. Representative photomicrographs of H\&E- and Masson's trichrome-stained kidney sections are shown in Fig. 1. The kidney tissues from untreated CKD rats showed severe interstitial fibrosis, tubular atrophy and dilation, and glomerulosclerosis. Compared with the untreated group, the FC-treated group exhibited modest improvements in the severity of tubulointerstitial lesions and glomerulosclerosis.

Tissue Iron Distribution. Data are shown in Fig. 2. Compared with the control and placebo-treated CKD rats, the FC-treated CKD rats showed significant increase in stainable iron in the proximal tubular epithelial cells of the remnant kidney and epithelial and subepithelial segments of the descending colon. However, no significant difference was observed in the stainable iron in the liver or proximal jejunum between the three groups.

Inflammatory, Oxidative, Fibrosis, and Nrf2 Data. The remnant kidney tissue in $\mathrm{CKD}$ rats exhibited a significant upregulation in nuclear translocation of $\mathrm{p} 65$ subunit of $\mathrm{NF}-\kappa \mathrm{B}$ (3.2-fold), pointing to activation of NF- $\kappa \mathrm{B}$, the master regulator of the inflammatory pathway. This was accompanied by significant increases in proinflammatory and reactive oxygen, nitrogen, and halogen species-generating molecules, including MCP-1 (5.1-fold), iNOS (1.8-fold), COX-2 (2.4-fold), gp91phox (1.9-fold), NOX-4 (2.0-fold), and MPO (2.4-fold) and accumulation of nitrotyrosine (2.1-fold). Inflammatory and oxidative pathways activation in the kidneys of the untreated CKD animals was accompanied by significant upregulations of TGF- $\beta$ (4.7-fold), PAI-1 (8.1-fold), and $\alpha$-SM actin (9.0-fold), reflecting the fibrotic pathway activation (Figs. 3-5). This was accompanied by significant reduction of the nuclear Nrf2 translocation (0.6-fold) and reduced expression of its key target genes, including catalase (0.4-fold), glutathione peroxidase (0.4-fold), Cu/Zn-SOD (0.3-fold), heme oxygenase-1 

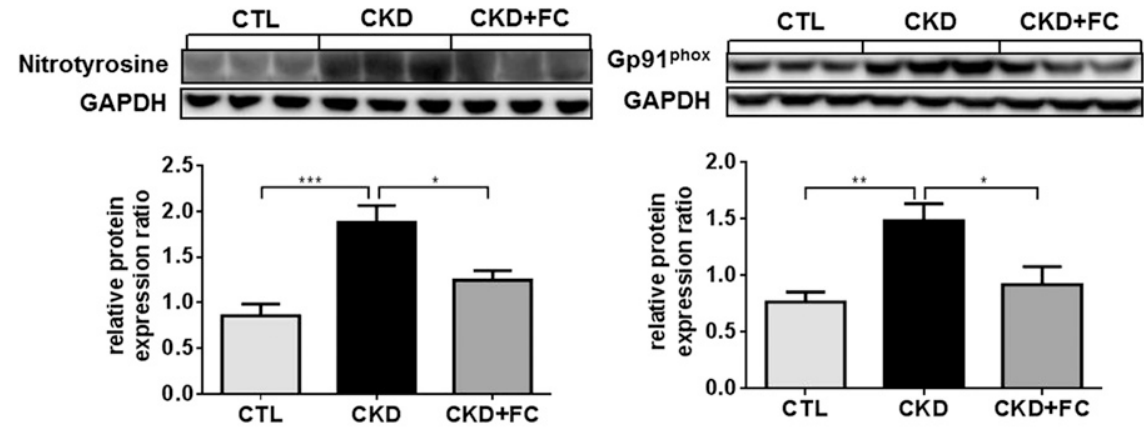

Fig. 3. Impact of treatment with $\mathrm{FC}$ on expression of reactive oxygen, halogen, and nitrogen speciesproducing pathways. Representative Western blots and group data demonstrating protein abundance of $\mathrm{NAD}(\mathrm{P}) \mathrm{H}$ oxidase subunits (NOX-4 and gp91phox), nitrotyrosine, and MPO in the renal tissues of CTL and CKD- and CKD+FC-treated animals. Data are CTL CKD CKD+FC

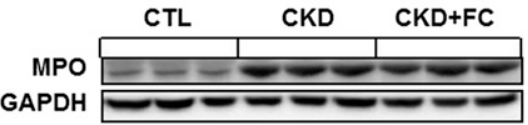
mean \pm S.E.M. $(n=9) . * P<0.05$; $* * P<0.01 ; * * P<<$

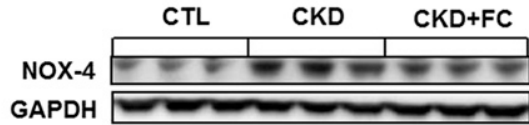
GAPDH 0.001 . GAPDH was used as a loading control for cytoplasmic extracts.
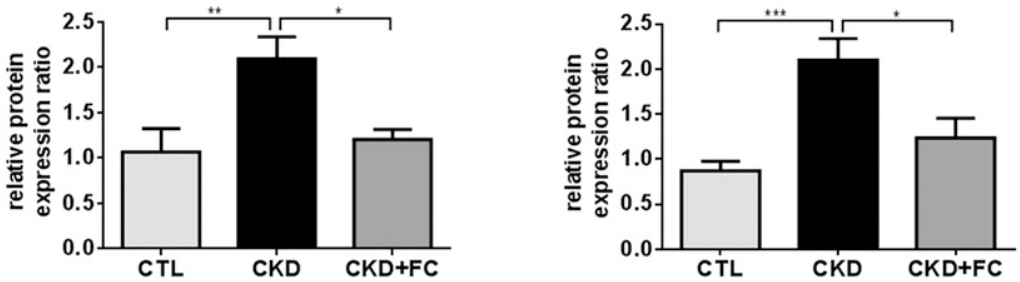

(0.2-fold), and eNOS (0.6-fold) (Fig. 6). These findings are in line with our earlier studies (Kim and Vaziri, 2010; Aminzadeh et al., 2012) and point to impairment of Nrf2 pathway, which plays a major role in the pathogenesis of the CKD-associated oxidative stress and inflammation.
FC treatment partially attenuated the severity of upregulations of several inflammatory, oxidative, and profibrotic molecules, including MCP-1 (0.7-fold), COX-2 (0.6-fold), gp91phox (0.6-fold), NOX-4 (0.6-fold), iNOS (0.4-fold), MPO (0.6-fold), PAI-1 (0.5-fold), TGF- $\beta$ (0.5-fold), and $\alpha$-SM actin
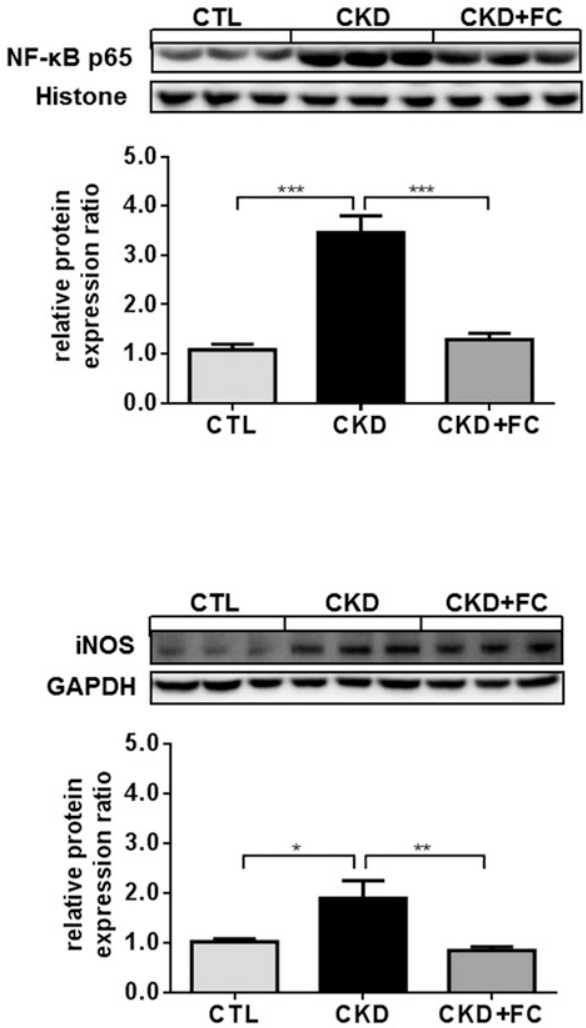
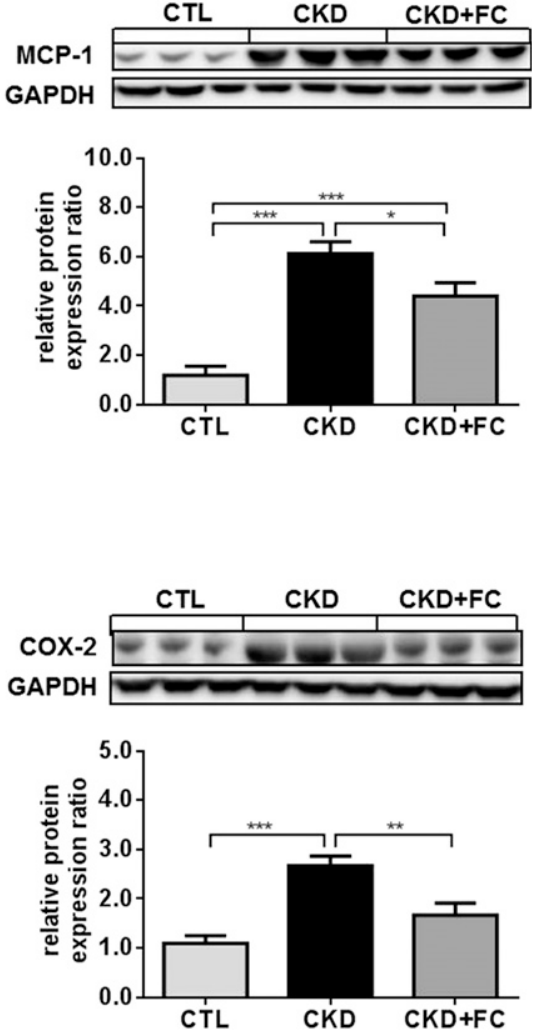

Fig. 4. Impact of treatment with $\mathrm{FC}$ on inflammatory pathway. Representative Western blots and group data demonstrating the nuclear content of p65 active subunit of NF- $\kappa \mathrm{B}$, and protein abundance of MCP-1, iNOS, and COX-2 in the renal tissues of CTL and CKD- and CKD+FC-treated animals. Data are mean \pm S.E.M. $(n=9) . * P<0.05 ; * * P<0.01 ; * * P<$ 0.001 . Histone was used as a loading control for nuclear extracts. GAPDH was used as a loading control for cytoplasmic extracts. 

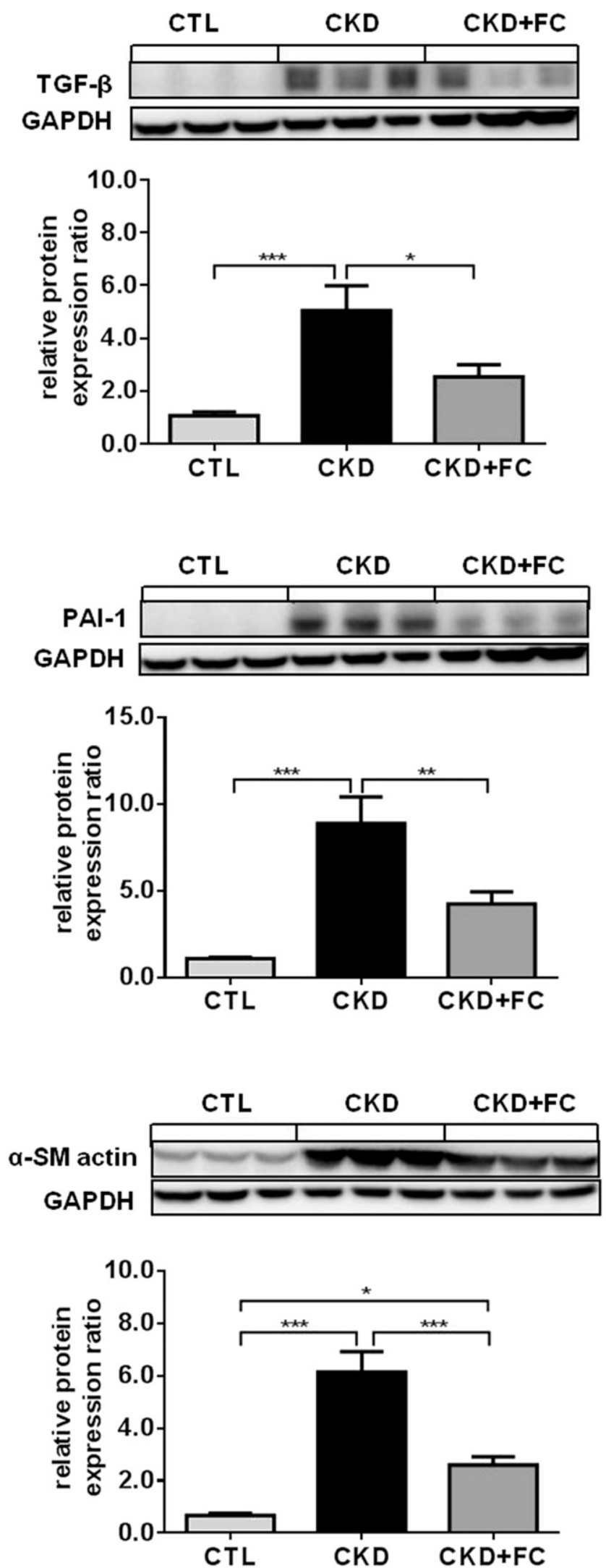

Fig. 5. Impact of treatment with $\mathrm{FC}$ on fibrosis pathway. Representative Western blots and group data demonstrating TGF- $\beta$, PAI- 1 , and $\alpha$-SM actin abundance in the renal tissues of CTL and CKD- and CKD+FCtreated animals. Data are mean \pm S.E.M. $(n=9)$. $* P<0.05 ; * * P<0.01$ $* * * P<0.001$. GAPDH was used as loading control for cytoplasmic extracts. (0.4-fold). This was associated with partial improvements in Nrf2 (1.4-fold) activity and expression of several antioxidant and cytoprotective molecules, including $\mathrm{Cu} / \mathrm{Zn}$-SOD (2.6-fold), catalase (1.7-fold), and eNOS (1.7-fold). Taken together, the partial improvements of inflammatory, oxidative, fibrosis, and Nrf2 pathways can account for the modest attenuation of the tubulointerstitial lesions and glomerulosclerosis in the FC-treated CKD group.

Colonic Epithelial Junction Complex Data. Compared with the normal control group, the CKD rats showed significant depletion of the ZO-1, occludin, and JAM-1 proteins in the colon. The FC-treated CKD group exhibited restoration of ZO-1 abundance but no significant change in the occludin or JAM-1 protein abundance (Fig. 7).

\section{Discussion}

In contrast to conventional oral iron products, ferric citrate significantly increases serum ferritin, transferrin saturation, and hemoglobin levels in ESRD patients and reduces their IV iron and ESA requirements (Block et al., 2015; Lewis et al., 2015; Tanaka et al., 2016; Fishbane et al., 2017). Consistent with the results of the clinical trials, administration of FC resulted in reduction of serum phosphate and the rise in serum iron and hemoglobin levels in our CKD rats. This was associated with modest improvements in renal function and partial attenuation of glomerulosclerosis and tubulointerstitial lesions. To explore the underlying mechanisms of the salutary effects of the given dosage of FC on renal histology in CKD animals, we examined its impact on the expression of inflammatory, oxidative, and fibrotic pathways that mediate CKD progression. The results revealed partial attenuation of CKD-induced upregulations of oxidative, inflammatory, and fibrosis pathways in the remnant kidney of the treated CKD rats. This was associated with a modest improvement in the nuclear translocation of Nrf2 and expression of its cytoprotective antioxidant enzymes/proteins. These findings indicated the safety and efficacy of the given dosage of $\mathrm{FC}$ in the CKD animals. In fact, administration of FC for 6 months in a group of Japanese hemodialysis patients, whose serum ferritin levels were kept below $100 \mathrm{ng} / \mathrm{ml}$, resulted in ameliorations of anemia and iron deficiency and reduced erythropoietin requirement without raising the plasma biomarkers of oxidative stress or inflammation (Tanaka et al., 2016). The observed reduction of ESA resistance in patients treated with ferric citrate is consistent with amelioration of inflammation and oxidative stress that induce ESA resistance (Vaziri et al., 2016a).

Intravenous iron preparations are administered at doses that far exceed the plasma transferrin binding capacity, leading to appearance of catalytically active iron in the circulation, formation of hydroxyl radical, and amplification of oxidative stress through the Fenton reaction. This phenomenon can, in turn, lead to adverse consequences by intensifying the CKD-related inflammation and oxidative stress (Salahudeen et al., 2001; Tovbin et al., 2002; Lim and Vaziri, 2004a; Guz et al., 2006; Kuo et al., 2008). Owing to the slow and gradual nature of intestinal iron absorption, this phenomenon can be avoided with the proper dosage of oral FC, as shown in the recent study enrolling Japanese hemodialysis patients (Tanaka et al., 2016). However, as shown by Umanath et al. (2015) and noted in the accompanying editorial 

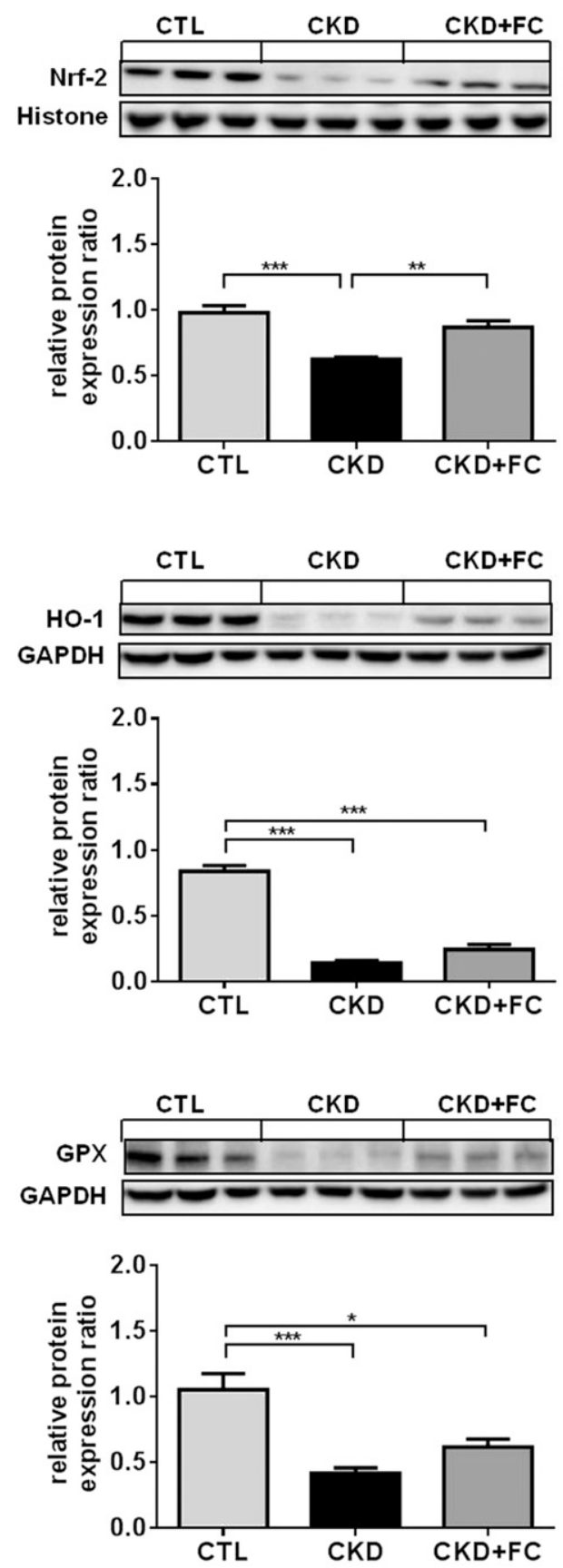
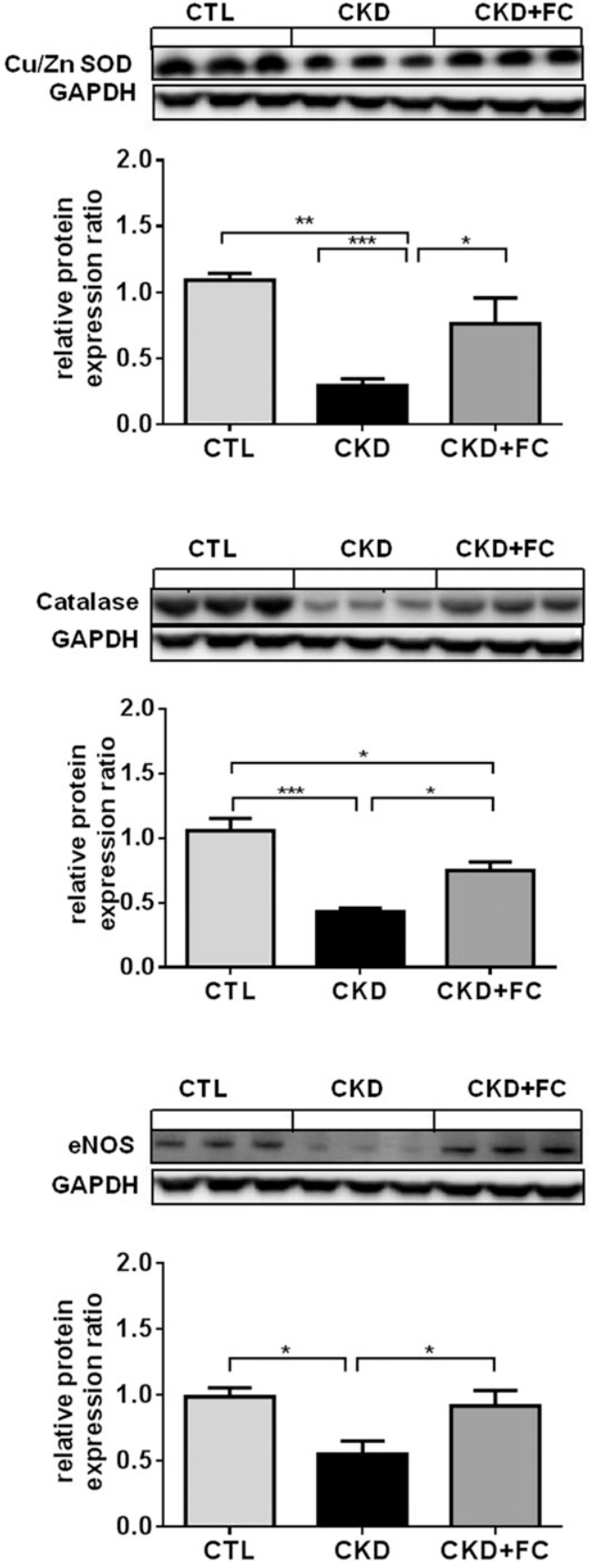

Fig. 6. Impact of treatment with FC on Nrf2 pathway. Representative Western blots and group data depicting nuclear translocation of Nrf2 and protein abundances of its downstream gene products $\mathrm{Cu} / \mathrm{Zn}$-SOD, catalase, glutathione peroxidase (GPX), heme oxygenase-1 (HO-1), and eNOS in the renal tissues of CTL and CKD- and CKD+FC-treated animals. Data are mean \pm S.E.M. $(n=9$ rats $)$. $* P<0.05 ; * * P<0.01 ; * * * P<0.001$. Histone was used as a loading control for nuclear extracts. GAPDH was used as loading control for cytoplasmic extracts.
(Qunibi, 2015), long-term and excessive dosage of ferric citrate can result in iron overload. Therefore, iron indices should be carefully monitored and FC administration discontinued when iron levels exceed the acceptable limits.

Several factors contribute to the pathogenesis of anemia in patients with advanced CKD and ESRD, including erythropoietin resistance and deficiency, shortened erythrocyte life span, and elevated hepcidin, which impairs intestinal absorption of iron and its release from the storage sites. Intestinal absorption of iron, which is mediated by divalent metal transporter and ferroportin in duodenum and proximal jejunum, is tightly regulated and is inhibited by hepcidin (Zhao et al., 2013). Owing to systemic inflammation, hepcidin level is significantly elevated in patients and animals with advanced
CKD (Wang and Babitt, 2016). Owing to impaired iron absorption, most ESRD patients are routinely treated with intravenous iron products. However, oral administration of FC has been reported to expand iron stores and lower the need for parenteral iron products in the CKD and ESRD populations (Block et al., 2015; Lewis et al., 2015; Tanaka et al., 2016; Fishbane et al., 2017). To our knowledge the mechanism and site of iron absorption with consumption of the phosphate binder FC is unknown. However, the microbial community in the colon is known to convert indigestible carbohydrates such as cellulose to short-chain fatty acids that represent a major nutrient for colonocytes (Wong et al., 2006). Therefore, a large insoluble complex such as FC could serve as a substrate for colonic microbial flora that enable its breakdown to single FC 

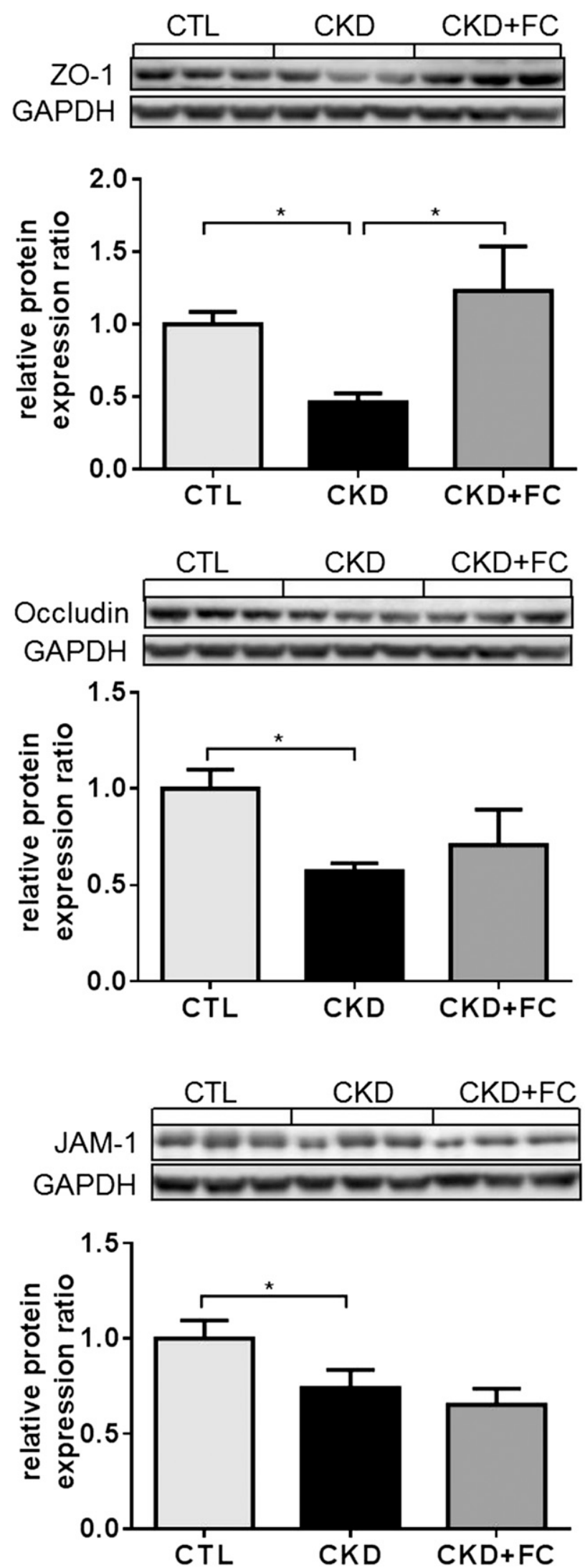

Fig. 7. Impact of treatment with $\mathrm{FC}$ on the epithelial junctional complex proteins ZO-1, occludin, and JAM-1 in the colon tissues of CTL and CKDand CKD+FC-treated animals. Data are mean \pm S.E.M. $(n=9) . * P<0.05$. Histone was used as a loading control for nuclear extracts. GAPDH was used as loading control for cytoplasmic extracts. molecules. Uptake of citrate through short-chain fatty acid transporters by colonic epithelial cells can be followed, in turn, by uptake of iron to maintain electrical neutrality. This phenomenon may account for atypical iron absorption in the reported FC-treated CKD patients (Block et al., 2015; Lewis et al., 2015; Tanaka et al., 2016; Fishbane et al., 2017) and CKD rats shown in our study, as a bypass of the normal route of iron absorption.

Earlier studies demonstrated marked depletion of the protein constituents of colonic epithelial tight junction and its contribution to the uremia-associated systemic inflammation (Vaziri et al., 2012, 2013b,c). To explore the underlying mechanism of amelioration of inflammation in the FC-treated $\mathrm{CKD}$ rats, we measured the key components of colonic epithelial junction complex in the study rats. In confirmation of the above studies and compared with the normal control group, the CKD rats showed marked depletion of the ZO-1, occludin, and JAM-1 proteins in the wall of colon. The FC-treated group showed restoration of $\mathrm{ZO}-1$ abundance, the intracellular protein that serves as the anchor of the transcellular components of the epithelial tight junction complex. Therefore, reversal of its deficiency may have partly contributed to the observed attenuation of inflammation in the FC-treated CKD group.

In conclusion administration of the novel phosphate binder ferric citrate resulted in modest amelioration of renal function and histology and partial improvements in inflammation, oxidative stress, and fibrosis in the remnants kidneys of $\mathrm{CKD}$ rats.

\section{Authorship Contributions}

Participated in research design: Jing, Vaziri.

Conducted experiments: Jing, Nunes, Khazaeli, Lau.

Performed data analysis: Jing, Nunes, Farzaneh.

Wrote or contributed to the writing of the manuscript: Jing, Vaziri.

\section{References}

Agarwal R, Kusek JW, and Pappas MK (2015) A randomized trial of intravenous and oral iron in chronic kidney disease. Kidney Int 88:905-914.

Aminzadeh MA, Sato T, and Vaziri ND (2012) Participation of endoplasmic reticulum stress in the pathogenesis of spontaneous glomerulosclerosis-role of intra-renal angiotensin system. Transl Res 160:309-318.

Block GA, Fishbane S, Rodriguez M, Smits G, Shemesh S, Pergola PE, Wolf M, and Chertow GM (2015) A 12-week, double-blind, placebo-controlled trial of ferric citrate for the treatment of iron deficiency anemia and reduction of serum phosphate in patients with CKD Stages 3-5. Am J Kidney Dis 65:728-736.

Brookhart MA, Freburger JK, Ellis AR, Wang L, Winkelmayer WC, and Kshirsagar AV (2013) Infection risk with bolus versus maintenance iron supplementation in hemodialysis patients. J Am Soc Nephrol 24:1151-1158.

Eschbach JW, Egrie JC, Downing MR, Browne JK, and Adamson JW (1987) Correction of the anemia of end-stage renal disease with recombinant human erythropoietin. Results of a combined phase I and II clinical trial. $N$ Engl J Med 316: 73-78.

Ferrari P, Kulkarni H, Dheda S, Betti S, Harrison C, St Pierre TG, and Olynyk JK (2011) Serum iron markers are inadequate for guiding iron repletion in chronic kidney disease. Clin J Am Soc Nephrol 6:77-83.

Fishbane S, Block GA, Loram L, Neylan J, Pergola PE, Uhlig K, and Chertow GM (2017) Effects of ferric citrate in patients with nondialysis-dependent CKD and iron deficiency anemia. J Am Soc Nephrol 28:1851-1858.

Garcia-Fernandez N, Echeverria A, Sanchez-Ibarrola A, Páramo JA, and ComaCanella I (2010) Randomized clinical trial on acute effects of i.v. iron sucrose during haemodialysis. Nephrology (Carlton) 15:178-183.

Guz G, Glorieux GL, De Smet R, Waterloos MA, Vanholder RC, and Dhondt AW (2006) Impact of iron sucrose therapy on leucocyte surface molecules and reactive oxygen species in haemodialysis patients. Nephrol Dial Transplant 21:2834-2840.

Kalantar-Zadeh K, Streja E, Miller JE, and Nissenson AR (2009) Intravenous iron versus erythropoiesis-stimulating agents: friends or foes in treating chronic kidney disease anemia? Adv Chronic Kidney Dis 16:143-151.

Kim HJ and Vaziri ND (2010) Contribution of impaired Nrf2-Keap1 pathway to oxidative stress and inflammation in chronic renal failure. Am J Physiol Renal Physiol 298:F662-F671.

Kuo KL, Hung SC, Wei YH, and Tarng DC (2008) Intravenous iron exacerbates oxidative DNA damage in peripheral blood lymphocytes in chronic hemodialysis patients. J Am Soc Nephrol 19:1817-1826. 
Lewis JB, Sika M, Koury MJ, Chuang P, Schulman G, Smith MT, Whittier FC, Linfert DR, Galphin CM, Athreya BP, et al.; Collaborative Study Group (2015) Ferric citrate controls phosphorus and delivers iron in patients on dialysis. $J \mathrm{Am}$ Soc Nephrol 26:493-503.

Lim CS and Vaziri ND (2004a) The effects of iron dextran on the oxidative stress in cardiovascular tissues of rats with chronic renal failure. Kidney Int 65:1802-1809.

Lim CS and Vaziri ND (2004b) Iron and oxidative stress in renal insufficiency. Am J Nephrol 24:569-575.

Matsuo A, Iida A, Tanimoto M, Matsushita M, and Miyamoto K (2014) The utility of the phosphate binder, ferric citrate hydrate (JTT-751), about phosphorus absorption-reducing effect in normal rats. Ren Fail 36:1291-1297.

McFarlane SI, Chen SC, Whaley-Connell AT, Sowers JR, Vassalotti JA, Salifu MO, Li S, Wang C, Bakris G, McCullough PA, et al.; Kidney Early Evaluation Program Investigators (2008) Prevalence and associations of anemia of CKD: Kidney Early Evaluation Program (KEEP) and National Health and Nutrition Examination Survey (NHANES) 1999-2004. Am J Kidney Dis 51(4 Suppl 2):S46-S55.

Pai AB, Boyd AV, McQuade CR, Harford A, Norenberg JP, and Zager PG (2007) Comparison of oxidative stress markers after intravenous administration of iron dextran, sodium ferric gluconate, and iron sucrose in patients undergoing hemodialysis. Pharmacotherapy 27:343-350.

Qunibi WY (2015) Is it too much of a good thing? A new era in phosphate binder therapy in ESRD. J Am Soc Nephrol 26:2311-2313.

Sakurai H, Hisada Y, Ueno M, Sugiura M, Kawashima K, and Sugita T (1996) Activation of transcription factor NF-kappa B in experimental glomerulonephritis in rats. Biochim Biophys Acta 1316:132-138.

Salahudeen AK, Oliver B, Bower JD, and Roberts LJ, II (2001) Increase in plasma esterified F2-isoprostanes following intravenous iron infusion in patients on hemodialysis. Kidney Int 60:1525-1531.

Tanaka M, Miyamura S, Imafuku T, Tominaga Y, Maeda H, Anraku M, Yamasaki K, Kadowaki D, Ishima Y, Watanabe H, et al. (2016) Effect of a ferric citrate formulation, a phosphate binder, on oxidative stress in chronic kidney diseases-mineral and bone disorder patients receiving hemodialysis: a Pilot study. Biol Pharm Bull 39:1000-1006.

Tovbin D, Mazor D, Vorobiov M, Chaimovitz C, and Meyerstein N (2002) Induction of protein oxidation by intravenous iron in hemodialysis patients: role of inflammation. Am J Kidney Dis 40:1005-1012.

Umanath K, Jalal DI, Greco BA, Umeukeje EM, Reisin E, Manley J, Zeig S, Negoi DG, Hiremath AN, Blumenthal SS, et al.; Collaborative Study Group (2015) Ferric citrate reduces intravenous iron and erythropoiesis-stimulating agent use in ESRD. J Am Soc Nephrol 26:2578-2587.

Vaziri ND (2012) Epidemic of iron overload in dialysis population caused by intravenous iron products: a plea for moderation. Am J Med 125:951-952.

Vaziri ND, Kalantar-Zadeh K, and Wish JB (2016a) New options for iron supplementation in maintenance hemodialysis patients. Am J Kidney Dis 67:367-375.

Vaziri ND, Ni Z, Oveisi F, Liang K, and Pandian R (2002) Enhanced nitric oxide inactivation and protein nitration by reactive oxygen species in renal insufficiency. Hypertension 39:135-141.

Vaziri ND, Wong J, Pahl M, Piceno YM, Yuan J, DeSantis TZ, Ni Z, Nguyen TH, and Andersen GL (2013a) Chronic kidney disease alters intestinal microbial flora. Kidney Int 83:308-315.

Vaziri ND, Yuan J, Nazertehrani S, Ni Z, and Liu S (2013b) Chronic kidney disease causes disruption of gastric and small intestinal epithelial tight junction. Am $J$ Nephrol 38:99-103.

Vaziri ND, Yuan J, and Norris K (2013c) Role of urea in intestinal barrier dysfunction and disruption of epithelial tight junction in chronic kidney disease. Am J Nephrol 37:1-6.

Vaziri ND, Yuan J, Rahimi A, Ni Z, Said H, and Subramanian VS (2012) Disintegration of colonic epithelial tight junction in uremia: a likely cause of CKDassociated inflammation. Nephrol Dial Transplant 27:2686-2693.

Vaziri ND, Zhao YY, and Pahl MV (2016b) Altered intestinal microbial flora and impaired epithelial barrier structure and function in CKD: the nature, mechanisms, consequences and potential treatment. Nephrol Dial Transplant 31: $737-746$.

Wang CY and Babitt JL (2016) Hepcidin regulation in the anemia of inflammation. Curr Opin Hematol 23:189-197.

Wong JM, de Souza R, Kendall CW, Emam A, and Jenkins DJ (2006) Colonic health: fermentation and short chain fatty acids. J Clin Gastroenterol 40:235-243.

Zhao N, Zhang AS, and Enns CA (2013) Iron regulation by hepcidin. J Clin Invest 123:2337-2343.

Address correspondence to: Dr. Nosratola D. Vaziri, Division of Nephrology and Hypertension, University of California, Irvine Medical Center, Orange, CA 92868. E-mail: ndvaziri@uci.edu; or Dr. Wanghui Jing, School of Pharmacy, Xi'an Jiaotong University, NO. 76 Yanta west road, Xi'an, Shaanxi, China. E-mail: jingwanghui1987@163.com 